\title{
LA REPÚBLICA DEL SABER EN JAQUE. IGUALDAD Y SUJETO EN LA ENSEÑANZA DE LA FILOSOFÍA
}

\author{
Leonardo Javier Colella* \\ Rocío Díaz Salazar**
}

\begin{abstract}
RESUMEN
La intención del presente artículo es reconstruir teóricamente el concepto de "subjetivación" en la enseñanza de la filosofía, a partir del vínculo entre la teoría del sujeto de Alain Badiou y el postulado de la "igualdad de las inteligencias" de Jacques Rancière. El nuevo escenario educativo, producto del vínculo igualitario entre maestro y alumno, es interpretado como una disrupción del estado de las relaciones pedagógicas de las instituciones modernas. Este "acontecimiento" constituye la posibilidad para la construcción de un sujeto que sostenga (y a la vez, se sostenga en) aquello que trastorna el orden de las cosas. Este proceso de subjetivación hace posible un escenario de enseñanza de la filosofía que no implique únicamente la reproducción de saberes establecidos, sino que inaugure una vía posible para la intervención creadora del "filosofar".
\end{abstract}

Palabras clave: Subjetivación. Relación maestro-alumno. Igualdad de las inteligencias. Acontecimiento Jacotot. Repetición creativa.

\begin{abstract}
The aim of this paper is to theoretically reconstruct the concept of "subjectivation" in the teaching of philosophy, from the link between the theory of the subject of Alain Badiou and the postulate of the "equality of intelligences" of Jacques Rancière. The new educational setting, product of the equitable relationship between teacher and student, is interpreted as a disruption of the status of the educational relations of
\end{abstract}

\footnotetext{
* Becario doctoral del Consejo Nacional Científico y Técnico (CONICET). Profesor Universitario de Filosofía por la Universidad Nacional de General Sarmiento (UNGS). E-mail: leonardojcolella@yahoo.com.ar

** Licenciada en Ciencias de la Comunicación Social por la Facultad de Ciencias Sociales, Universidad de Buenos Aires. E-mail: rocbelds@hotmail.com
} 
the modern institutions. This event is the possibility for the construction of a subject that holds (and at the same time, that is holding in) what upsets the order of things. This subjectivation process allows a scenario of teaching philosophy that has not exhaust just in the reproduction of established knowledge, but open one path to the creative intervention of "philosophize".

Keywords: Subjectivation. Teacher-student relationship. Equality of intelligence. Jacotot event. Creative repetition.

\section{Repetición y creación en la enseñanza de la filosofía}

En un artículo denominado Philosophy as creative repetition, el filósofo Alain Badiou propone una tesis en la que describe a la filosofía como una forma de "repetición creativa" (BADIOU, 2007). En ella distingue esencialmente dos tendencias acerca de la naturaleza de la filosofía. La primera de ellas, la interpreta como un conocimiento reflexivo: conocimientos sobre la verdad y los valores en los ámbitos teoréticos y prácticos, respectivamente. La forma que vehiculiza el desarrollo de esta tendencia es la de la explicación escolar, entendida como método de transmisión de aquellos saberes reflexivos. La segunda postura concibe a la filosofía como una acción: una transformación subjetiva, una conversión radical. En este caso, la filosofía es delimitada por la singularidad de un acto, que Badiou asocia con la acusación ejercida contra Sócrates de "corromper a los jóvenes", cuyo rasgo distintivo sería impulsar el ejercicio de la crítica y la revuelta por sobre la imitación y la obediencia. Esta segunda tendencia, que interpreta a la filosofía como una actitud antes que como un conocimiento, no se enseña mediante la explicación escolar, sino que se vive o se experimenta con otros, al modo en que Sócrates dialogaba con los jóvenes en las calles de Atenas.

En este sentido, la idea de "repetición creativa", aplicada al desarrollo de la filosofía, describe la existencia de un acto que es formalmente común a todas las corrientes filosóficas. En éstas, lo invariable sería el gesto de división que constituye el acto filosófico: la distinción con respecto a otras filosofías y demás saberes; y la reorganización de las experiencias teoréticas y prácticas a partir de esas nuevas divisiones normativas. La 
filosofía se hallaría delimitada por el insistente dinamismo entre lo que afirma y lo que cuestiona, por la relación tensional entre un ejercicio crítico de afirmación, oposición y creación. A diferencia de la ciencia, en la que sus propias novedades reemplazan las teorías anteriores, ella se constituye a partir de ese permanente afirmar y refutar. Según Badiou, en la filosofía siempre existirá una intención de reconstituir lo existente partiendo de disposiciones normativas nuevas: se trata de poner de relieve el lazo entre el ser y el acontecer, lo que conforma el rasgo propio de la "repetición creativa".

Este gesto crítico, que se reitera a través de los diferentes filósofos, se lleva a cabo bajo determinadas condiciones variables: lo que cambia es el contexto histórico. Cuando el acto filosófico se enfrenta a la actualidad específica de cada contexto se produce una nueva creación intelectual. El acto filosófico se ejerce, según Badiou, luego del despliegue de algunos acontecimientos en política, ciencia, arte, amor. Estos acontecimientos son su condición de posibilidad y, a la vez, proporcionan la necesidad de impulsar un cambio a través de aquel invariante acto filosófico. En tal sentido, el autor afirma que la filosofía dependería de algunos campos nofilosóficos que serían sus "condiciones".

Recapitulando, lo que hay de repetición en la filosofía es el gesto crítico del acto filosófico, y lo que hay de creación es impulsado por el nuevo contexto específico en el que se desarrolla dicho acto. Es por eso que el autor sugiere el concepto de "repetición creativa" para definir a la filosofía.

Alejandro Cerletti propone emplear los conceptos de "repetición" y "creación", aplicados por Badiou a la filosofía misma, para el caso de la enseñanza y el aprendizaje filosóficos (CERLETTI, 2008a). En el proceso de enseñanza y aprendizaje intervendrían dos aspectos articulados entre sí: una dimensión "objetiva", que incluiría los saberes preestablecidos, las obras filosóficas, las investigaciones históricas, etc.; y una "subjetiva", que estaría constituida por la novedad de aquellos que despliegan la actitud filosófica, por su apropiación y recreación de los problemas filosóficos. Estos diferentes aspectos estarían presentes en el interior del aula durante el desarrollo de una clase. En ella, los problemas planteados apuntan en dos direcciones: hacia la universalidad del saber filosófico y hacia la singularidad del sujeto de aprendizaje. 
Cerletti (2008b, p. 88) destaca el concepto de "pensamiento" utilizado por Badiou para caracterizar la toma de posición subjetiva frente a los saberes preestablecidos. Pensar sería, en este sentido, interceder, atravesar y reconstruir los saberes que enuncian lo que hay; el pensamiento constituiría una ruptura con respecto a los saberes enciclopédicos que representan un estado de cosas dado.

La denominada enseñanza tradicional se agota en la transmisión de los saberes filosóficos acumulados en su propia historia, dirección que asume el único sentido de profesor a estudiante. En tal aspecto, la enseñanza de la filosofía no trascendería las fronteras de la dimensión "objetiva". Sin embargo, el "filosofar" sobrepasa los límites de la simple repetición, ya que adopta como parte constitutiva de sí mismo el gesto crítico que permite el despliegue de alguna novedad.

Para que la enseñanza de la filosofía haga propio el ejercicio de una "repetición creativa", se debe asumir la posibilidad de "hacer" filosofía al mismo tiempo que se la enseña y se la aprende. El matiz creativo sería posible en la medida que exista una intervención subjetiva que permita que los saberes preestablecidos sean revisados en el contexto específico de la clase. De este modo, maestro y alumno comenzarían a "filosofar", lo que implicaría que ellos mismos desplieguen una repetición creativa en tanto desarrollen el gesto filosófico de la crítica de lo establecido en su propio contexto y desde su propia mirada particular.

Si se pretende enseñar a "filosofar" es preciso suponer la necesidad de lograr un cambio subjetivo en la forma de conceptualizar la propia realidad. Esto requerirá un singular proceso de subjetivación en la enseñanza de la filosofía, ya que quienes filosofan se reapropian, de una manera novedosa, de las preguntas y saberes filosóficos, y lo hacen desde su particular mirada. Si bien la enseñanza de la filosofía actúa en articulación con sujetos individuales y se apoya en factores objetivos, involucra una construcción subjetiva en sentido colectivo. El sujeto constituido en el ámbito educativo parte de un pensar dialógico compartido, en el que confluyen las miradas problematizadoras particulares. En este aspecto, el sujeto no sería el profesor o el alumno, sino el vínculo construido por (y entre) ambos. 


\section{Sujeto y amor en la enseñanza de la filosofía}

La teoría del sujeto de Alain Badiou se encuentra inscripta en su análisis general del ser y el acontecer, desarrollado principalmente en Théorie du sujet (1982), L'être et l'événement (1988) y Logiques des mondes (2006). La constitución de un sujeto está íntimamente relacionada con un "acontecimiento", concepto que el autor utiliza para designar la disrupción de un estado de normalidad de las situaciones existentes y de la regularidad de los saberes instituidos que las tornaban inteligibles. A esta construcción que reúne y sujeta los efectos de un acontecimiento y que decide sostener, impulsar y ser coherente con aquello que trastorna el orden de una situación, Badiou la denomina sujeto. Y utiliza el concepto de fidelidad para designar aquella decisión de sostener y ser consecuente con la disrupción de un estado de cosas. Es decir, las activaciones subjetivas son promovidas por un acontecimiento y sustentadas a través de un procedimiento de fidelidad.

La constitución del sujeto no depende meramente del ser; surge de una estructura pero la atraviesa, precisa de un suplemento azaroso que Badiou designa acontecimiento. Se deviene sujeto cuando el individuo es transportado más allá de sí mismo por la fuerza de un acontecimiento. En tal aspecto, Badiou se aparta de Althusser y Lévy-Strauss pero también de Sartre: el sujeto no es constituido ni constituyente, es un surgimiento; es el sustento de una verdad que se genera en la situación a raíz de las consecuencias de un acontecimiento.

El concepto de verdad desarrollado por Badiou no se erige como un absoluto proveedor de sentido o como una verdad trascendente, sino que constituye una ruptura inmanente en tanto rastro acontecimiental propio de una situación. Una verdad atraviesa los saberes de una situación, fuerza los conocimientos establecidos del estado dominante de las cosas. Según Badiou, existirían al menos cuatro ámbitos en los que se producen acontecimientos y procedimientos de verdad: la política, el arte, la ciencia y el amor.

La educación no está contemplada por el autor como un ámbito independiente de producción de verdades. Más bien, podríamos suponer que la educación institucionalizada recogería las verdades de esos campos (científico, artístico, político, amoroso) y operaría a favor de una 
recomposición de esos ámbitos transformando aquellas verdades (inscriptas en situaciones particulares y desplegadas al interior de un acontecimiento) en saberes institucionalizados. La institución educativa se apropia de los enunciados verdaderos de los acontecimientos originados en cada campo y los transmite como parte de un saber instituido. Desde esta perspectiva de análisis, la educación podría ser estudiada a partir de cada uno de los procedimientos de verdad mencionados. Así podrían analizarse, por ejemplo, los aspectos relacionados con el amor (acontecimiento, verdad, sujeto, etc.) en la educación ${ }^{1}$.

En Éloge de l'amour (BADIOU, 2009), el autor plantea la constitución de un sujeto amoroso a partir del encuentro contingente entre dos personas, la construcción de un nuevo mundo desde un punto de vista descentrado respecto de uno mismo, la experiencia de ese mundo a partir de la diferencia y no únicamente de la identidad. A ese encuentro azaroso que evade la ley de ordenamiento de las cosas le otorga el estatuto de acontecimiento. En el amor, Badiou afirma que existiría la experiencia del traspaso de la singularidad pura a un orden de valor universal: que va desde lo particular de un encuentro hasta la experiencia de un mundo habitado desde la diferencia y no sólo desde la uniformidad.

El amor contiene, además del primer momento asociado al encuentro, una duración, una obstinación o fidelidad que el sujeto sostiene en el tiempo. Es decir, para la construcción subjetiva se requiere, además del encuentro acontecimiental, una fidelidad que sostenga aquella disrupción elemental del curso normal de las cosas.

El amor, para Badiou, es un "procedimiento de verdad" (como lo es también la política, el arte y la ciencia). La verdad, en el ámbito del amor, es la posibilidad de una nueva vivencia del mundo experimentado a partir de lo Dos, de la diferencia; es la prueba de que el mundo puede ser vivido de otro modo que mediante una conciencia individual. Lo universal en el amor consistiría en la experimentación de lo que es ser Dos. Este amor en el escenario de la enseñanza de la filosofía nos permitiría indagar

1 Para un análisis de los conceptos "acontecimiento" y "sujeto" políticos en la educación, puede consultarse Repetición, novedad y sujeto en la educación. Un análisis filosófico y político (CERLETTI, 2008b). 
sobre la posibilidad de una experiencia del filosofar desde un punto de vista descentrado de uno mismo, y ya no como un mero traspaso de los resultados de un filosofar ajeno.

Para el caso de la relación maestro-alumno, podríamos pensar si es posible un vínculo que permita desplegar un mundo a partir del prisma de la diferencia que constituya un único sujeto, en analogía con el Sujeto del amor enunciado por Badiou. Asimismo, podríamos considerar la posibilidad de un sujeto amoroso en la educación que fuerce el advenimiento de ese nuevo mundo, y que éste no sólo sea utilizado como material transferible desde una conciencia particular a otra. En sentido estricto, el amor no es simplemente un encuentro sino, además, una construcción, ya no desde una perspectiva unilateral, sino desde una múltiple. De este modo, podemos afirmar en consonancia con la teoría de Badiou, que el acontecimiento Jacotot $^{2}$ (RANCIÈRE, 1987), que propone un nuevo vínculo igualitario entre maestro y alumno al desconvenir con el estado de las relaciones pedagógicas de las instituciones educativas modernas, hace posible la construcción de un sujeto en la educación.

La relación institucional entre profesor y estudiante que critica Rancière a través de la figura de Jacotot está mediada por la tarea explicativa. La lógica de la "explicación" promueve dos consecuencias problemáticas al interior del espacio de la enseñanza de la filosofía: por un lado, al tratarse de la transferencia e incorporación de conocimientos filosóficos ya dados, reproduce estos saberes sin trascender la dimensión objetiva, relegando el acto filosófico y su dimensión creativa por fuera del ámbito de la enseñanza y el aprendizaje; por otro lado, al partir de una desigualdad que intenta subsanar (la disparidad entre los conocimientos del maestro y del alumno), obtura la construcción subjetiva de una experiencia colectiva al dividir las partes vinculadas afirmando la capacidad intelectual de una por sobre la incapacidad "provisoria" de la otra.

Sin embargo, la enseñanza de la filosofía, entendida como repetición

2 El acontecimiento Jacotot refiere a un conjunto de experiencias educativas del pedagogo Joseph Jacotot analizadas por Rancière en Le maître ignorant (1987), que básicamente representan la posibilidad de una nueva forma de vínculo entre maestro y alumno, en el que se abandona la lógica de la transmisión de saberes. Más adelante será abordado descriptivamente. 
creativa, requiere la construcción de un nuevo vínculo entre maestro y alumno que supere la lógica de la "explicación", ya que ésta postergaría la intervención subjetiva del gesto filosófico al consistir simplemente en un proceso gradual de transmisión de saberes. Si se considera a la filosofía principalmente como una actividad, la enseñanza de la filosofía no podría quedar circunscripta a la construcción de un vínculo maestro-alumno determinado por la asimetría de conocimientos y cuyo objetivo principal fuera la explicación. Por el contrario, la enseñanza de la filosofía implicaría trascender la dimensión objetiva de los saberes acumulados en su historia, y desplegar ella misma un nuevo vínculo que afirme la igualdad entre maestro y alumno en referencia al "filosofar".

En el marco de la teoría del sujeto de Alain Badiou, que demanda para la construcción subjetiva la doble instancia del acontecimiento y la fidelidad, la propuesta de la "igualdad de las inteligencias" planteada por Rancière, abre un nuevo modo de relación entre maestro y alumno, por lo que asume la posibilidad de una disrupción elemental en el contexto de las situaciones educativas. Este nuevo escenario configura la enseñanza y el aprendizaje filosóficos como una posición superadora del ámbito de reproducción de los saberes prefigurados por su historia (la enciclopedia) y del problema de la reapropiación actualizada de ellos a partir de los problemas del presente (el pensamiento).

\section{Igualdad y acontecimiento en la enseñanza de la filosofía}

Le Maître ignorant. Cinq leçons sur l'émancipation intellectuelle (RANCIÈRE, 1987) se construye a partir de las experiencias pedagógicas de la figura de Joseph Jacotot, que a comienzos del siglo XIX y por circunstancias azarosas provocaron en él un cambio de perspectiva con respecto a la enseñanza tradicional. Jacotot se vio forzado a abandonar Francia y a dictar clases de literatura francesa en una universidad de los Países Bajos. Él no hablaba holandés y sus estudiantes no comprendían el francés. A través de una edición bilingüe de Telémaco de Fenelón, sus alumnos, poco a poco, comparando página por página, renglón por renglón, palabra por palabra, no sólo terminaron por comprender el texto completo, sino que realizaron ensayos críticos que contenían valiosas observaciones 
personales sobre la obra, expresados en el nuevo idioma. A ésta, se sumaron muchas otras experiencias en las que Jacotot lograba exitosamente que sus alumnos aprendieran temáticas que él no sabía. El replanteo pedagógico elaborado por Rancière-Jacotot consideraba la posibilidad de aprender sin las explicaciones de un maestro (lo que no significaba prescindir de la figura del maestro, sino renunciar a su función de "transmisor" o "explicador").

La lógica de la explicación supone un proceso de transmisión de ciertos saberes que el maestro posee y que el alumno no y, consecuentemente, demanda cierta subordinación intelectual que configura un escenario de segmentación jerárquica entre poseedor y desposeído. Rancière señala que las instituciones educativas modernas han adoptado el rol de reproducir esta desigualdad jerárquica hasta el punto de convertirla en su propia condición de posibilidad: de tal forma que el sentido de la escuela acabe por convertirse en la perpetua designación de desiguales-inferiores, cuyo criterio diferenciador sería la carencia de ciertos saberes y su característica distintiva la incapacidad de obtenerlos por medio propio. La función esencial del maestro explicador sería la inscripción de quienes aprenden en un circuito dominado por estructuras estamentales y por una lógica de superiores e inferiores.

A través de la experiencia de Jacotot, Rancière sugiere que es posible pensar en la construcción de una nueva relación entre maestros y alumnos que no esté mediada por el recurso de la explicación. El maestro emancipador no es quien transmite su propio saber, sino quien, a través del vínculo entre voluntades, enseña al otro a ejercer su propia inteligencia. El conocimiento pierde la única dirección, antes desplegada desde el maestro hacia el alumno, para configurar nuevos y múltiples sentidos. Los saberes no constituyen un universo diferenciador (entre aquél que los posee y aquellos que no), sino que, por el contrario, asumen la forma de interrogantes compartidos que sostienen el vínculo entre el maestro y el alumno, en el que se verifica la igualdad de las inteligencias.

El nuevo vínculo entre maestro y alumno iniciado a partir del acontecimiento Jacotot, no se erige con la finalidad de reducir aquello que el aprendiz ignora mediante la transmisión de contenidos, sino que lo hace construyendo un nuevo mundo a partir de la observación, el pensamiento y la acción de ambos, lo que podría desplegar un proceso educativo de 
subjetivación que se sostenga en una experiencia de pensamiento colectivo y no en la constatación de la desigualdad de las inteligencias que instituye la lógica de la transmisión de saberes.

La propuesta de Rancière, lejos de apuntar a la constatación de una desigualdad e intentar progresivamente a futuro disuadirla mediante la transferencia de contenidos, postula la igualdad como punto de partida para extraer de ella toda la potencia de sus consecuencias. En efecto, no se trata de corroborar la carencia de ciertos conocimientos, sino de instar al descrédito de la inferioridad de las inteligencias. Desde una perspectiva rancieriana, la inteligencia no es interpretada como la posesión de ciertos saberes o como el ejercicio de ciertas facultades intelectivas complejas, sino más bien, como la capacidad de pensar y decidir sobre la propia vida a través de la posibilidad de indagarse a uno mismo y poner en cuestión la relación que se tiene con los saberes. Esta variante de carácter gnoseológico permite afirmar que la igualdad de las inteligencias, en vez de ser un objetivo a conquistar mediante la explicación, debe ser un postulado filosófico-político inicial para toda propuesta de enseñanza que se pretenda filosófica.

En Ecole, production, égalité (RANCIÈRE, 1988), un texto publicado posteriormente a El maestro ignorante, el autor intenta describir el escenario en el que el nuevo vínculo "igualitario" sería posible. Para ello realiza un análisis histórico de la Francia postrevolucionaria que le permite identificar y abstraer la forma-escuela y sustraerla de varios aspectos constitutivos de las instituciones educativas modernas. La formaescuela no es definida por una finalidad social externa, sino que asume la fisonomía de una forma simbólica que justamente establece una separación con los tiempos, espacios y ocupaciones sociales. La forma-escuela asume una particular distribución de los tiempos, ya que no se constituye en el lugar "exclusivo" para el aprendizaje, sino que es definida principalmente por la figura del ocio. La scholé separa dos usos del tiempo: el que se utiliza forzadamente para la producción y el que se dispone libremente al estar dispensado de las exigencias del trabajo. Por ello, la scholé griega delimitaba el modo de vida de los iguales, hacía de los escolares de la Academia o del Liceo los iguales por excelencia.

Así, la forma-escuela tomaría distancia con respecto a las necesidades del universo laboral y a la transmisión de contenidos proveedores de 
herramientas útiles para la vida productiva. En este sentido, este aspecto formal que Rancière destaca de la scholé desestimaría la hipótesis de la escuela como una institución de distribución de las ciencias cuyas consecuencias serían la justicia distributiva. En contraposición a ello, la forma-escuela constituiría un posible escenario de igualdad al relegar a los "escolares" del mundo social desigual e incluirlos en el mismo tiempo de ocio. La escuela moderna ha olvidado que no es un medio para conquistar, eventualmente a futuro, una presunta igualdad (ya que ninguna igualdad futura es garantizada a través de la transferencia de ciertos saberes), sino que ella misma alberga, formalmente, un espacio-tiempo igualitario en el que todos pueden "aprender por aprender", potencia que se halla interrumpida por el mismo afán desigualitario del modelo "explicador".

La escuela, para Rancière, no sería el lugar de promoción de una ciencia útil para la vida social, sino más bien, un espacio privilegiado para la verificación de la igualdad, y por lo tanto, sus eventuales consecuencias transformadoras irrumpirían de la distancia que disponga con respecto a la lógica del universo explicativo y productivo.

Precisamente, lo que interesaba a Jacotot era verificar esta igualdad. De este modo, cuando enseñaba francés en Lovaina se proponía enseñar algo más a sus estudiantes que una lengua extranjera. Incluso enseñó hebreo a un hijo "discapacitado mental" de su impresor, pero tampoco lo realmente importante para él era el hebreo. No se trataba de un método para explicar la ciencia al pueblo, se trataba de un anuncio a la vez intrépido y provocador: cualquier hombre puede lo que puede otro hombre. Jacotot no hizo más que dedicarse a proclamar lo que el azar lo llevó a descubrir: la igualdad de las inteligencias.

El mundo de la ilustración tuvo que oírlo. La conmoción fue más allá de Lovaina, Bruselas y La Haya, atravesó París y Lyon, se expandió por Europa hacia el norte y hacia el este, lo oyeron en Inglaterra y en Prusia, traspasó al imperio ruso y sus ecos alcanzaron el continente americano. Durante dos décadas, la República del Saber sufrió un cimbronazo en sus raíces al momento de su propia fundación (RANCIÈRE, 1987, p. 34).

Pero para mediados del siglo XIX, el acontecimiento Jacotot fue perdiendo vigor. La fuerza de la industria, la República, la opinión pública y la prensa requirieron un método acelerado para la instrucción de las masas: un amplio sistema de enseñanza graduado, nacional y profesional. 
Demandaron, asimismo, métodos expeditivos para enseñar, entre otras cosas, a leer: la explicación. Y así el progresismo ilustrado reactivaba el viejo principio de la desigualdad de las inteligencias (Ibídem, p. 161).

Pero la voz disonante de Jacotot, desplegada en aquel momento histórico clave, se erige en la actualidad como testimonio de otra vía posible para la educación. El nuevo escenario educativo, delimitado a raíz del vínculo igualitario entre maestro y alumno, constituye una disrupción del estado de las relaciones pedagógicas de las instituciones modernas. Este acontecimiento abre la posibilidad para la construcción de un sujeto que sostenga (y a la vez, se sostenga en) aquello que trastorna el orden de las cosas. Este sujeto supone el desarrollo de un pensamiento compartido que despliegue una actitud creadora y crítica respecto de los antiguos saberes. En la enseñanza de la filosofía como repetición creativa, maestro y alumno devienen un único sujeto del filosofar, en el que afirman, verifican y actualizan el postulado de la igualdad de las inteligencias.

\section{Referências}

BADIOU, Alain. Éloge de l'amour. Paris: Flammarion, 2009. . L'être et l'événement. París: Seuil, 1988.

. Logiques des mondes. L'être et l'événement, 2. París: Seuil, 2006. . Philosophy as creative repetition. The Symptom, United States, v. 8, Winter, 2007.

. Théorie du sujet. París: Seuil, 1982.

CERLETTI, Alejandro. La enseñanza de la filosofía como problema filosófico. Buenos Aires: Libros del Zorzal, 2008a.

. Repetición, novedad y sujeto en la educación. Buenos Aires: Del Estante Editorial, 2008b.

RANCIÈRE, Jacques. Ecole, production, égalité. In: L'école de la démocratie. Edilig: Fondation Diderot, 1988.

. Le Maître ignorant. Cinq leçons sur l'émancipation intellectuelle. París: Fayard, 1987.

Data de registro: 11/09/2012

Data de aceite:25/01/2013 